\title{
THE HANDBOOK FOR NURSES
}

Ir is hoped that this Handbook will be published early in November, 1908, by Messrs. Baillière, Tindall and Cox.

\section{COMPILATION FORMS AND EXPANSION TABLES.}

THE Compilation Forms and Expansion Tables, which have been found very necessary in the preparation of Asylum Statistics on the new system sanctioned by the Association, have been in general use during this year. A few errors and inconveniences have been reported. If any member has found any particular inconvenience he can kindly send a note of it either to Messrs. Adlard and Son or to the General Secretary.

\section{OBITUARY.}

\section{Dr. John Cameron.}

WE regret to record the death of Dr. John Cameron, Medical Superintendent of the Argyll and Bute Asylum at Lochgilphead. He was born in Killin 67 years ago, and qualified at Edinburgh University. His first appointment was to Crichton Royal Institution, Dumfries, from which he went to the Argyll and Bute Asylum in 1874 , in succession to Dr. James Rutherford. He was at one time an ardent volunteer, and retired from the Argyll and Bute Artillery with the rank of Lieutenant-Colonel. About three months ago Dr. Cameron went to Edinburgh, where he died, suffering from an internal malady, no hope being then entertained of his recovery.

\section{NOTICES BY THE REGISTRAR.}

Examination for the Nursing Certificate.

List of the successful candidates at the examination for the Nursing Certificate held in May, 1908.

ENGLAND.

Birmingham City (Rubery Hill).-Males: George Hy. Cushan, George T. Mason.

Bethlem Hospital.-Male : Edward Cole. Females: Eva F. Scott, Isabella Evans, Harriett Mann, Alice M. Fosbery.

Canterbury Borough.-Male : Frederick T. Stannard. Female: Hilda J. Thornycroft.

Caterham._-Males: Harold J. Edwards, James Payne, Albert Atkins. Females : May Pringle, Mary McConnell.

Hull City.-Males: Thomas France, Arthur W. Legard, Albert Clabby. Females : Rose Stott, Lydia Thornton, Mary M. Moore, Hephzibah Harp.

Leavesden.-Males : Ashley A. Haseldine, George Roberts, Frederick Rhodes. Females : Mary E. Norwell, Mary Sullivan.

Newcastle City.-Females : Louisa Denham, Grace Thompson.

Notts City.-Male : Walter E. Cooper.

Retreat, York.-Females : Eliza J. Brearley, Ethel Gordon Dunbar, Geraldine S. McKew.

Sunderland Borough.-Male : Fred. A. McCullagh.

Storthes Hall.-Female : Gertrude Green.

Scalebor Park.-Males : William Atkinson, Frederick C. Horton, Harry Gilder, George Cawood, John Henry Cawood. Females : Mary Jane Stewart, Mary Charlotte Walls. 
St. Luke's Hospital.-Female : Esther H. Savage.

Private nurse examined at Long Grove.-Female: Helen Armstrong.

Private nurse examined at Claybury.-Male : John Mahony.

Bristol City.-Males : Timothy Rowan, Frederick W. Bolland, Arthur Legg,

Henry Castle. Females : May Jackson, Helen F. Wigglesworth, Florence E. Button.

Cumberland and Westmorland.-Male : Sidney Hill. Females: Edith Hayden, Mary Farquhar, Gertrude Jamison.

Derby County. - Male : Thomas Gamble. Female; M. H. O'Bierne.

Devon County.-Males: Edwin Harding, Frederick S. Eager, George

Whitehouse. Females: Beatrice Edworthy, Elizabeth A. Morgan, Ada Saunders, Bessie Simons.

Essex County.-Females : Frances Lily Penny, Anna M. Lewsey, Alma Bale,

Maria J. Jones.

Herts County.-Male: Ernest J. Haywood. Female : Annie Alden.

Kent County (Maidstons).-Males: Percy F. Laming, Frank Neve, Guy K.

Knight, Jesse Collar, Donald P. White. Females : Susanna Shaw, Rose Kemsley,

Emily Mankelow, Alice M. Franklin, Ellen McCormick.

Tooting.-Females : Elizabeth Jordan, Ethel Wicks.

Salop County.-Female : Elizabeth A. Haywood.

Lancaster.-Males: David G. Edmonds, Herbert Ordish, James H. Paxton,

Charles Tiplady, Joseph Lomax, Robert Parkin, William Power. Females : Annie

Towlson, Lily Ada Tidyman, Mary Hurtley, Margaret Norris, Frances $M$.

Parkinson, Mary Preston, Julia Brough, Mary Keating.

Lancaster County (Winwick).-Males: William E. Milligan, John Thompson,

James William Scaling, Martin Meehan. Females: Jane Elizabeth Forbes,

Edith A. Bennison, Annie Duckett, Annie R. Laurie.

Lancaster County (Whittingham).-Males : Edward Seed, Ernest Pittaway,

James Fisher, Arthur Hy. Fann, Charles Dean, Frederick Coupe, Charles

Broadley, Gill Akroyd, Albert Sutcliffe, George E. Gillett, William Allison, Willie

Riley, John Richardson, Louis C. Patmore, Arthur L. Packer, Thos. Wm. Nelson,

R. Hy. Holding, John Farrington. Females : Mary McGann, Jessie Fisher, Clara

Smith, Marion Snow, Bridget Regan, Rose Owen, Louisa S. Gibson, Mary J.

Harkin, Ellen Annie Cox.

Middlesex County (Napsbury).-Male : Robert Morris. Females : Ada Gamble,

Jeanie Dumble, Margaret McCarthy, Frances M. Simmonds, Florence E. Pettit,

A. A. E. McKnight.

Norfolk County.-Male : Henry Nobbs. Females : Sarah A. Fisher, Henrietta

M. Gallant, Winifred Jones.

Somerset and Bath (Wells).-Male: Samuel J. Heath.

Staffs County (Cheddleton).-Females: Sarah C. Achurch, Maud E. G. R.

Smith.

Suffolk County (Melton).-Males: Charles Addison, Henry Fuller, Cornelius

Courtney. Female : Rosetta Clare.

Surrey County (Brookwood).-Males: Rodger Howard, John D. Howden,

William Marshall. Female : Margaret Hiney.

Sussex County (Chichester).-Females: Winifred B. Brooks, Winifred M.

Brown, Ada H. Frith, Florence E. Godfrey, Esther Johnson, Laura L. King, Edith

M. Yeadon.

Sussex County (Hellingly).-Males : James Marks Braids, Septimus C.

Somerville, William M. Fells.

Three Counties (Hitchin).-Males : James Moore, William Parsons. Females:

Dorothy B. Brown, May Ludford, Lucy Lambert.

Barnsley Hall.-Male: Joseph Grundy. Females : Mary Healey, Annie Best.

Yorks (Wakefield).-Males : Francis L. Jackson, George Hutchinson, Henry T.

Lilliman. Females: Annie Quickfall, Nellie Corridon, Lily Scales, Christiana

Powles, Annie Tennant, Alice M. Astbury, Emma Hasker.

Yorks (Wadsley).-Female : Clara Vaughan.

London County (Bexley).-Males : Alfred Doidge, James Spittles. Females :

Jennie E. Crombie, Jane L. Hunter, Eliza Johnson, May Seaward.

London County (Cane Hill). - Male : James Dye.

London County (Claybury).-Males : William James Hall, Charles C. Saye, 
James William White. Females: Kate Brenton, Ellen Walsh, Anna Tinney, Lucy M. M. Gale, Frederica Doyle, Elizabeth Peglar, Florence E. Thorpe, Margaret Mewitt, Louisa Hamilton, Elizabeth Todd, Kathleen Mary Nelson, Charlotte E. Briody.

London County (Colney Hatch).-Females: Annie Vile, Daisy J. Munday, Eva Steele, Emma Ayling, Kate Hobbs, Lily E. Williams, Lizzie E. Humphreys, Agnes E. Hope, E. G. Bradley, Ada M. A. Dodd, Ada A. Drayton, Emily E. Newell, Sarah Ralph, Emma E. Robertson, Elizabeth V. Rose, Elizabeth Clarke, Louisa Moore, Hilda Wood, Louisa B. Adlington, Sarah L. Wheatley, Edith A.

Edwards, Annie Groves, Alice L. Child, Florence Hampson, Jane E. Frost,

Elizabeth A. Emerson, Hannah Hills, Amy Davies.

London County (Hanwell).-Females: Kate Williams, Sophia Farr, Cissie Gollidge, Nora E. Wooster, Grace Eyre.

London County (Horton).-Males: Hugh Johnston, William G. Randall, James Staddon, Adam S. Calder, Ernest S. Dean, Michael J. Oliver. Females: Agnes Kelly, Margaret A. Morrison, Florence Boulch, Euphemia Percival, Annie Newman, Eliza A. Johnston.

London County (Long Grove).-Males : Bertram H. Mitchell, Ernest R. Webber, Donald C. Clark, Frank M. M. Hardy, Samuel J. Coleman. Females: Beatrice Cawthron, Elizabeth Randle, Margaret Baird.

Private nurse examined at Bicton (Salop).-Female : Margaret K. Simmonds. Kent County (Chartham).-Male : Frederick Howlett. Females: Maud M. Mullins, Annie G. J. Easterbrook.

Chester County.-Males: Arthur Pritchard, Ernest A. Jones, Edward Owen, John Smith, Thomas Henry Newport, John P. Schofield. Females : Alice M. Glover, Catherine Valentine, E. M. Hargreaves, Daisy McIntosh, Harriet Valentine.

Warwick County.-Males : Austin Keen, Clarence Henry Lane. Females : Amy Sharp, Teresa Power.

\section{WALES.}

Glamorgan.-Males : Richard Jenkins, John R. Elmslie, Davie J. Thomas. Females: Elizabeth M. Davies, Margaret James, Blodwin Lewis, Sarah Lewis, Charlotte Missinden, Florence E. M. Skey, Mary Ann Owen.

Newport Borough.-Males : Albert Davenport, James R. Cook, Harry Bailey. Female : Evelyn H. Nigh.

Brecon and Radnor.-Male: W. G. Franklin.

\section{SCOTLAND.}

Aberdeen Royal.-Males : George Heron, James Duncan. Female : Mary Helen Minty.

Ayr District.-Male: Patrick McBarron. Female : Mary Lindsay.

Crichton Royal.-Male: Adam Lothian. Females : Jeannie Fordyce, Christina

Reid, Annabella Black, Mary Cameron, Elsie Macpherson.

Edinburgh Royal.-Females : Isabel J. K. Dott, Isabel K. Craigs, Annetta M. L. Ingram, Helen Hepburn, Margaret Alexander.

Fife and Kinross.-Males : John Adamson, Peter Scott Brown. Females : Annie Main, Margaret $\mathbf{H}$. Sharp.

Glasgow District (Lensie).-Females : Haslett Margaret, Mary Jenkins Smith, Jeanie G. Park, Mary McWilliam, Margaret Fleming, Mary M. Black, Margaret Symon.

Glasgow District (Gartloch).-Male: Temple C. Cormack. Females: Mary Pansy Allan, Marion K. Stevenson, Mary A. Wilson, Elizabeth Richardson, Ellen A. Cleary.

Glasgow District (Gartnavel).-Males : Alexander Ewing, Louis Jenner, Walter B. Lynas. Females : Isabella A. Helen, Bessie W. Thomson, Elizabeth Swanson, Margaret C. Robertson.

Govan District.-Male : Alexander Murdock. Female: Nettie S. McNish.

Inverness District.-Male : John M. Munro. Female : Agnes MacIntyre.

Kingseat.-Female : Elizabeth Robertson.

Lanark District.-Male : William Strachan. Females : Christina W. Greenhorn, Annie Bradley, Mary McNeil Weir, Jeanie Jackson. 
Montrose.-Males : John A. Thomson, John Nicoll. Females : Alice McKenzie, Annie C. Milne, Cecilia Fullerton, Annie Chapman, Charlotte Buchan.

Perth District.-Females : Ella MacKenzie, Annie F. Dewar, Margaret C. Boyd. Riccartsbar.-Male : James Simpson.

Roxburgh District.-Females: Helen McFarlane, Mary A. Jarvie.

Stirling Distriot.-Males: Martin Scanlan, John T. Mowat. Females : Alice E. Forster, Janet W. Marshall, Margaret Wards, Annie E. Mulholland, Margaret Lippiatt.

IRELAND.

Ballinasloe.-Males: William Hickey, Marks Kilalea, Timothy Churchill. Females : Mary Gorman, Nora Cullinan, Mary A. Finnerty.

Clonmel.-Males : Patrick Harding, James Grady. Females: Mary Maher, Mary Milton.

Cork District.-Males : Denis Mahony, John Coughlan, John Keane. Females : Annie Murphy, Minnie Reardon, Ellie O'Brien, Nora Motherway, Mary A. Sullivan, Anna Gould, Julia Lordon, Kate Twomey.

Downpatrick.-Males: John Barry, John Murtagh, Patrick King, William James

McConvey, William James Tuft, William James Hanna, Joseph Hodgson.

Enniscorthy.-Male: James Nolan. Female : Annie Walsh.

Londonderry.-Females : Margaret Jacob, Kathleen Murphy.

Monaghan.-Males : William Haine, Arthur Graham, Peter Murphy, Joseph

Johnston, Owen Connolly, Joseph Morrow, John Stewart, Patrick McKenna

Females : Eliza Jane Clarke, Mary Ann McAdam, Mary Anne Boyle, Bridget Holland.

Portrane.-Females : Mary Kieran, Mary McGuirk, Anne Maria Orr, Kate Henneberry, Sarah Jane Taylor, Mary F. Nolan, Mary Phelan.

Richmond.-Males: John Pallis, John Brennan, Lawrence Murray, Michael

Boland, John Quinn, William McDonald. Females : Edith Casey, Mary Aungier,

Helena K. O'Beirne, Mary A. Breen, Margaret Alice Hartford, Maria McGuiness,

Elizabeth O'Callaghan.

Waterford.-Males : James McDonald, Thomas J. Dunn. Females : Catherine

Maher, Mary B. O'Toole.

St. Patrick's Hospital.-Female: Katherine S. D. Graham.

The following is a list of the questions which appeared on the paper:

1. What is meant by reflex action? Give three examples.

2. What points should a nurse attend to in the general management of a patient suffering from heart disease ?

3. Describe the mental features seen in "epileptic insanity."

4. What are the special risks in cases of senile insanity, and how should they be guarded against $P$

5. A patient spits bright red blood. Where is it likely to come from, and how would you treat the patient till the doctor comes?

6. Describe the performance of artificial respiration.

7. What is a convulsion? In what diseases are convulsions most likely to occur?

8. What are the precautions to be observed in nursing patients suffering from consumption ?

9. What are the principal conditions that lead to a progressive loss of weight ?

10. What would lead you to believe that a patient suffers from hallucinations of hearing? Illustrate your answer by the signs observed in two patients known to you. Why are hallucinations of grave importance in many cases?

\section{NURSING CERTIFICATE}

The next examination for the Nursing Certificate will take place on Monday, the 2nd November, 1908. 